\title{
The Dirichlet problem for the
} time-fractional advection-diffusion equation in a line segment

\author{
Yuriy Povstenko ${ }^{1 *}$ and Joanna Klekot ${ }^{2}$
}

\section{"Correspondence:}

j.povstenko@ajd.czest.pl

${ }^{1}$ Institute of Mathematics and

Computer Science, Jan Długosz

University, Częstochowa, 42-200, Poland

Full list of author information is

available at the end of the article

\begin{abstract}
The one-dimensional time-fractional advection-diffusion equation with the Caputo time derivative is considered in a line segment. The fundamental solution to the Dirichlet problem and the solution of the problem with a constant boundary condition are obtained using the integral transform technique. The numerical results are illustrated graphically.
\end{abstract}

MSC: $26 \mathrm{~A} 33 ; 45 \mathrm{~K} 05$

Keywords: fractional calculus; Caputo fractional derivative; non-Fickian diffusion; fractional advection-diffusion equation; Mittag-Leffler function

\section{Introduction}

The constitutive equation for the matter flux (see, for example, [1])

$$
\mathbf{j}=-a \operatorname{grad} c+\mathbf{v} c
$$

where $a$ denotes the diffusivity coefficient, $\mathbf{v}$ is the velocity vector, in combination with the balance equation for mass results in the standard advection-diffusion equation (under assumption $\mathbf{v}=$ const):

$$
\frac{\partial c}{\partial t}=a \Delta c-\mathbf{v} \cdot \operatorname{grad} c
$$

Equation (2) can be interpreted in terms of diffusion or heat conduction with additional velocity field as well as in terms as transport processes in porous media, Brownian motion or groundwater hydrology [1-6]. In the case of one spatial coordinate $x$, the advectiondiffusion equation (2) takes the form

$$
\frac{\partial c}{\partial t}=a \frac{\partial^{2} c}{\partial x^{2}}-v \frac{\partial c}{\partial x}
$$

In the last few decades, an increasing interest has been observed in the study of equations with derivatives of fractional order which have many applications in physics, geophysics, geology, rheology, engineering and bioengineering [7-14]. The time-nonlocal

(C) 2016 Povstenko and Klekot. This article is distributed under the terms of the Creative Commons Attribution 4.0 International License (http://creativecommons.org/licenses/by/4.0/), which permits unrestricted use, distribution, and reproduction in any medium, provided you give appropriate credit to the original author(s) and the source, provide a link to the Creative Commons license, and indicate if changes were made. 
generalizations of the constitutive equation (1) were analyzed in [15] (compare this analysis with that of the generalized Fourier or Fick law carried out in [14, 16-19]). In the case of the 'long-tail' power kernel, the generalized constitutive equation for the mass flux has the following form [15]:

$$
\mathbf{j}=D_{R L}^{1-\alpha}[-a \operatorname{grad} c(t)+\mathbf{v} c(t)]
$$

where $D_{R L}^{\alpha} c(t)$ is the Riemann-Liouville fractional derivative of the order $\alpha$ [20-22]:

$$
D_{R L}^{\alpha} c(t)=\frac{\mathrm{d}^{n}}{\mathrm{~d} t^{n}}\left[\frac{1}{\Gamma(n-\alpha)} \int_{0}^{t}(t-\tau)^{n-\alpha-1} c(\tau) \mathrm{d} \tau\right], \quad n-1<\alpha<n .
$$

Here $\Gamma(\alpha)$ is the gamma function. In combination with the balance equation for mass, the constitutive equation (4) leads to the time-fractional advection-diffusion equation

$$
\frac{\partial^{\alpha} c}{\partial t^{\alpha}}=a \Delta c-\mathbf{v} \cdot \operatorname{grad} c
$$

with the Caputo fractional derivative of the order $\alpha$ [20-22]:

$$
\frac{\mathrm{d}^{\alpha} c(t)}{\mathrm{d} t^{\alpha}}=\frac{1}{\Gamma(n-\alpha)} \int_{0}^{t}(t-\tau)^{n-\alpha-1} \frac{\mathrm{d}^{n} c(\tau)}{\mathrm{d} \tau^{n}} \mathrm{~d} \tau, \quad n-1<\alpha<n .
$$

A comprehensive survey of research on the fractional advection diffusion equation as well as of the numerical methods used for its solving can be found in [23]. In the literature there are only several papers in which the analytical solutions of fractional advections diffusion equation were considered [23-26]. In the present paper, we investigate the Dirichlet problem for equation (6) in a line segment $0<x<L$. Two types of boundary conditions are considered: the Dirac delta boundary condition for the fundamental solution and the constant boundary condition for the sought-for function.

\section{The fundamental solution to the Dirichlet problem}

We study the time-fractional advection-diffusion equation in a line segment $0<x<L$,

$$
\frac{\partial^{\alpha} c}{\partial t^{\alpha}}=a \frac{\partial^{2} c}{\partial x^{2}}-v \frac{\partial c}{\partial x}, \quad 0<\alpha \leq 1
$$

As usually $a>0, v>0,0<t<\infty$.

The advection diffusion equation (8) is considered under the zero initial condition

$$
t=0: \quad c(x, t)=0,
$$

and the Dirichlet boundary conditions at the ends of the segment:

$$
\begin{array}{ll}
x=0: & c(x, t)=g_{0} \delta(t), \\
x=L: & c(x, t)=0,
\end{array}
$$

where $\delta(t)$ is the Dirac delta function. The constant multiplier $g_{0}$ has been introduced to obtain the nondimensional quantity displayed in the figures. 
The new sought-for function

$$
c(x, t)=\exp \left(\frac{v x}{2 a}\right) u(x, t)
$$

reduces the considered initial-boundary-value problem to the following one:

$$
\begin{array}{ll}
\frac{\partial^{\alpha} u}{\partial t^{\alpha}}=a \frac{\partial^{2} u}{\partial x^{2}}-\frac{v^{2}}{4 a} u, \\
t=0: \quad u(x, t)=0, \\
x=0: \quad u(x, t)=g_{0} \delta(t), \\
x=L: \quad u(x, t)=0 .
\end{array}
$$

To solve the Dirichlet problem under examination, we use the finite sin-Fourier transform with respect to the spatial coordinate $x$. Such a transform is the convenient reformulation of the sin-Fourier series in the domain $0 \leq x \leq L$ :

$$
\begin{aligned}
& \mathcal{F}\{u(x)\}=\widetilde{u}\left(\xi_{k}\right)=\int_{0}^{L} u(x) \sin \left(\xi_{k} x\right) \mathrm{d} x, \\
& \mathcal{F}^{-1}\left\{\tilde{u}\left(\xi_{k}\right)\right\}=u(x)=\frac{2}{L} \sum_{k=1}^{\infty} \tilde{u}\left(\xi_{k}\right) \sin \left(\xi_{k} x\right),
\end{aligned}
$$

with

$$
\xi_{k}=\frac{k \pi}{L}, \quad k=1,2,3, \ldots
$$

The finite sin-Fourier transform of the second order derivative of a function is calculated according to the relation

$$
\mathcal{F}\left\{\frac{\mathrm{d}^{2} u(x)}{\mathrm{d} x^{2}}\right\}=-\xi_{k}^{2} \widetilde{u}\left(\xi_{k}\right)+\xi_{k}\left[u(0)-(-1)^{k} u(L)\right] .
$$

Application of the finite sin-Fourier transform (17) to equation (13) using (15), (16), and (20) leads to

$$
\begin{aligned}
& \frac{\partial^{\alpha} \widetilde{u}\left(\xi_{k}, t\right)}{\partial t^{\alpha}}=\left(-a \xi_{k}^{2}-\frac{v^{2}}{4 a}\right) \widetilde{u}\left(\xi_{k}, t\right)+a \xi_{k} g_{0} \delta(t), \\
& t=0: \quad \widetilde{u}\left(\xi_{k}, t\right)=0 .
\end{aligned}
$$

Next, we use the Laplace transform with respect to the time $t$. Recall that the Caputo fractional derivative for the Laplace transform requires the knowledge of the initial values of the function and its integer derivatives of the order $k=1,2, \ldots, n-1$ [20-22]:

$$
\mathcal{L}\left\{\frac{\mathrm{d}^{\alpha} u(t)}{\mathrm{d} t^{\alpha}}\right\}=s^{\alpha} u^{*}(s)-\sum_{k=0}^{n-1} u^{(k)}\left(0^{+}\right) s^{\alpha-1-k}, \quad 1<\alpha<n
$$

where $s$ is the transform variable. 
Applying the Laplace transform to equation (21) under the initial condition (22) gives

$$
\widetilde{u}^{*}\left(\xi_{k}, s\right)=a g_{0} \frac{\xi_{k}}{s^{\alpha}+a \xi_{k}^{2}+\frac{v^{2}}{4 a}} .
$$

Inversion of the integral transforms results in the solution

$$
u(x, t)=\frac{2 a g_{0} t^{\alpha-1}}{L} \sum_{k=1}^{\infty} \xi_{k} \sin \left(\xi_{k} x\right) E_{\alpha, \alpha}\left[-\left(a \xi_{k}^{2}+\frac{v^{2}}{4 a}\right) t^{\alpha}\right],
$$

where the formula [20-22]

$$
\mathcal{L}^{-1}\left\{\frac{s^{\alpha-\beta}}{s^{\alpha}+b}\right\}=t^{\beta-1} E_{\alpha, \beta}\left(-b t^{\alpha}\right)
$$

has been used with $E_{\alpha, \alpha}$ being the Mittag-Leffler function in two parameters $\alpha$ and $\beta$ :

$$
E_{\alpha, \beta}(z)=\sum_{n=0}^{\infty} \frac{z^{n}}{\Gamma(\alpha n+\beta)}, \quad \alpha, \beta>0
$$

Returning to the quantity $c(x, t)$ according to (12), we finally get the fundamental solution to the Dirichlet problem:

$$
c(x, t)=\frac{2 a g_{0} t^{\alpha-1}}{L} \exp \left(\frac{\nu x}{2 a}\right) \sum_{k=1}^{\infty} \xi_{k} \sin \left(\xi_{k} x\right) E_{\alpha, \alpha}\left[-\left(a \xi_{k}^{2}+\frac{v^{2}}{4 a}\right) t^{\alpha}\right] .
$$

Using the nondimensional quantities

$$
\bar{x}=\frac{x}{L}, \quad \bar{\xi}_{k}=L \xi_{k}=k \pi, \quad \bar{v}=\frac{v L}{a}, \quad \kappa=\frac{\sqrt{a} t^{\alpha / 2}}{L}, \quad \bar{c}=\frac{L^{2} c}{a g_{0} t^{\alpha-1}},
$$

we obtain

$$
\bar{c}(\bar{x}, \kappa)=2 \exp \left(\frac{\bar{v} \bar{x}}{2}\right) \sum_{k=1}^{\infty} \bar{\xi}_{k} \sin \left(\bar{\xi}_{k} \bar{x}\right) E_{\alpha, \alpha}\left[-\kappa^{2}\left(\bar{\xi}_{k}^{2}+\frac{\bar{v}^{2}}{4}\right) t^{\alpha}\right] .
$$

The fundamental solution (30) is shown in Figures 1-4 for different values of the order of fractional derivative $\alpha$, the time parameter $\kappa$, and the drift parameter $\bar{v}$.

\section{Constant boundary value of a function}

Next, we consider the time-fractional advection-diffusion equation (8) under zero initial condition (9) and the Dirichlet boundary condition with constant boundary values of the sought-for function:

$$
\begin{array}{ll}
x=0: & c(x, t)=c_{0}, \\
x=L: & c(x, t)=0 .
\end{array}
$$


Figure 1 Dependence of the fundamental solution (30) on distance $\bar{x}$ and the order $\alpha$ of fractional derivative $(\bar{v}=2, \kappa=0.2)$.

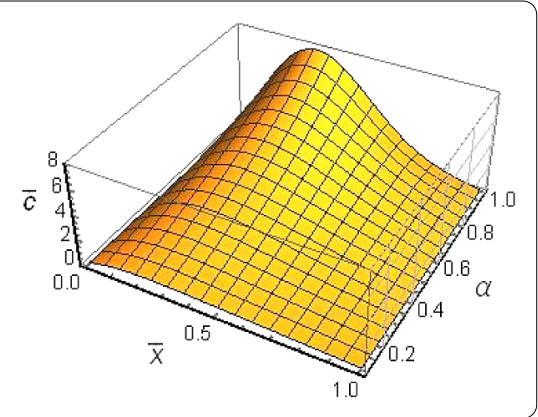

Figure 2 Dependence of the fundamental solution (30) on distance $\bar{x}$ and the order $\alpha$ of fractional derivative $(\bar{v}=2, \kappa=1)$.

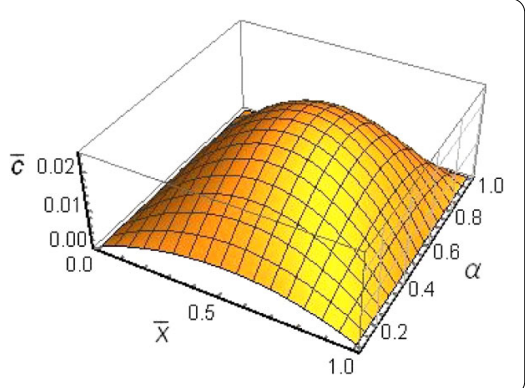

Figure 3 Dependence of the fundamental solution (30) on distance $\bar{x}$ and the time parameter $\kappa(\bar{v}=2, \alpha=0.5)$.

$\bar{c}$

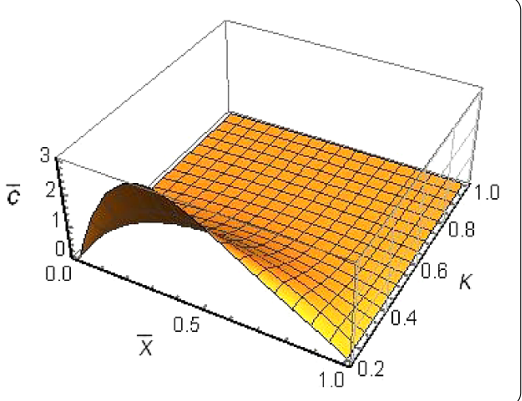

Figure 4 Dependence of the fundamental solution (30) on distance $\bar{x}$ and the drift parameter $\bar{v}(\alpha=0.5, \kappa=0.2)$.

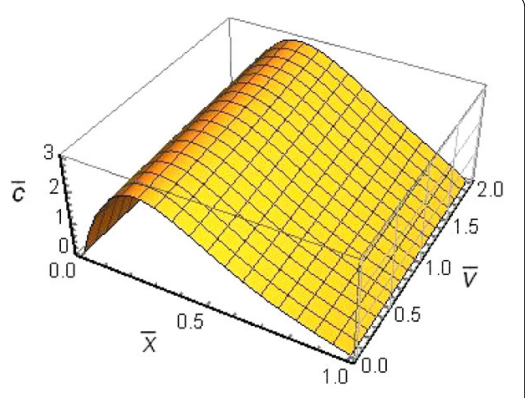

As above, the new function $u$ is introduced according to (12), and the Laplace transform with respect to time $t$ and the finite sin-Fourier transform with respect to the spatial coordinate $x$ give the solution in the transform domain:

$$
\tilde{u}^{*}\left(\xi_{k}, s\right)=a c_{0} \xi_{k} \frac{1}{s\left(s^{\alpha}+a \xi_{k}^{2}+\frac{v^{2}}{4 a}\right)} .
$$


Taking into account that

$$
\frac{1}{s\left(s^{\alpha}+a \xi_{k}^{2}+\frac{v^{2}}{4 a}\right)}=\frac{1}{a \xi_{k}^{2}+\frac{v^{2}}{4 a}}\left[\frac{1}{s}-\frac{s^{\alpha-1}}{s^{\alpha}+a \xi_{k}^{2}+\frac{v^{2}}{4 a}}\right]
$$

we obtain

$$
\tilde{u}^{*}\left(\xi_{k}, s\right)=\frac{a c_{0} \xi_{k}}{a \xi_{k}^{2}+\frac{v^{2}}{4 a}}\left[\frac{1}{s}-\frac{s^{\alpha-1}}{s^{\alpha}+a \xi_{k}^{2}+\frac{v^{2}}{4 a}}\right]
$$

or, after inversion of the integral transforms,

$$
u(x, t)=\frac{2 c_{0}}{L} \sum_{k=1}^{\infty} \frac{\xi_{k} \sin \left(\xi_{k} x\right)}{\xi_{k}^{2}+\frac{v^{2}}{4 a^{2}}}\left\{1-E_{\alpha}\left[-\left(a \xi_{k}^{2}+\frac{v^{2}}{4 a}\right) t^{\alpha}\right]\right\} .
$$

In this case $E_{\alpha}(z)$ is the Mittag-Leffler function in one parameter $\alpha$ :

$$
E_{\alpha}(z)=\sum_{n=0}^{\infty} \frac{z^{n}}{\Gamma(\alpha n+1)}, \quad \alpha>0 .
$$

Taking into account the following series [27]:

$$
\sum_{k=1}^{\infty} \frac{k \sin (k z)}{k^{2}+b^{2}}=\frac{\pi}{2} \frac{\sinh [(\pi-z) b]}{\sinh (\pi b)}
$$

and returning to the quantity $c(x, t)$ according to (12), we get

$$
\begin{aligned}
c(x, t)= & c_{0} \exp \left(\frac{v x}{2 a}\right)\left\{\frac{\sinh \left[\frac{\nu}{2 a}(L-x)\right]}{\sinh \left(\frac{\nu L}{2 a}\right)}\right. \\
& \left.-\frac{2}{L} \sum_{k=1}^{\infty} \frac{\xi_{k} \sin \left(\xi_{k} x\right)}{\xi_{k}^{2}+\frac{v^{2}}{4 a^{2}}} E_{\alpha}\left[-\left(a \xi_{k}^{2}+\frac{v^{2}}{4 a}\right) t^{\alpha}\right]\right\}
\end{aligned}
$$

and in the nondimensional form

$$
\begin{aligned}
\bar{c}(\bar{x}, \kappa)= & \exp \left(\frac{\bar{v} \bar{x}}{2}\right)\left\{\frac{\sinh \left[\frac{\bar{v}}{2}(1-\bar{x})\right]}{\sinh \left(\frac{\bar{v}}{2}\right)}\right. \\
& \left.-2 \sum_{k=1}^{\infty} \frac{k \pi \sin (k \pi \bar{x})}{(k \pi)^{2}+\frac{\overline{\bar{v}}}{4}} E_{\alpha}\left[-\kappa^{2}\left((k \pi)^{2}+\frac{\bar{v}^{2}}{4}\right)\right]\right\},
\end{aligned}
$$

where

$$
\bar{c}=\frac{c}{c_{0}},
$$

and the other nondimensional quantities are the same as in (29).

The solution (40) is presented in Figures 5-8 for different values of the order of fractional derivative $\alpha$, the time parameter $\kappa$, and the drift parameter $\bar{\nu}$. 


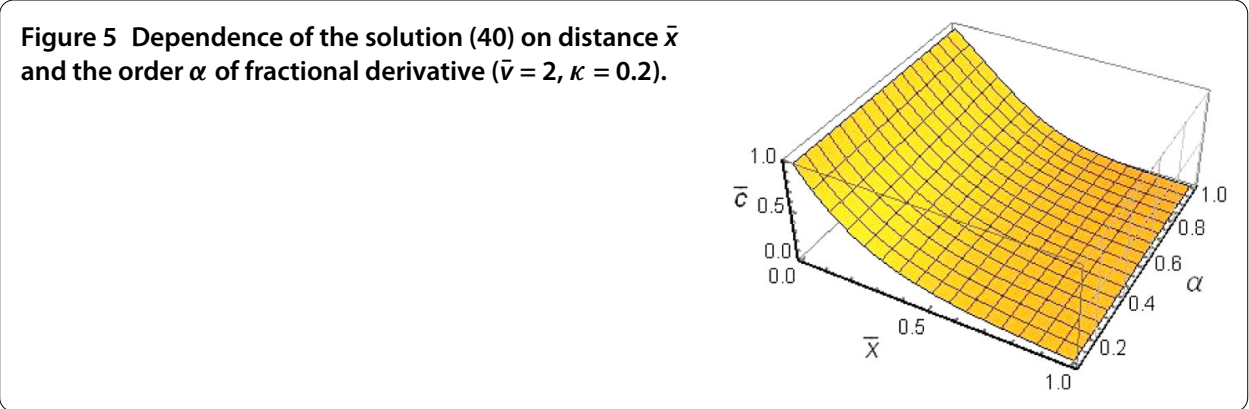

Figure 6 Dependence of the solution (40) on distance $\bar{x}$ and the order $\alpha$ of fractional derivative $(\bar{v}=2, \kappa=1)$.

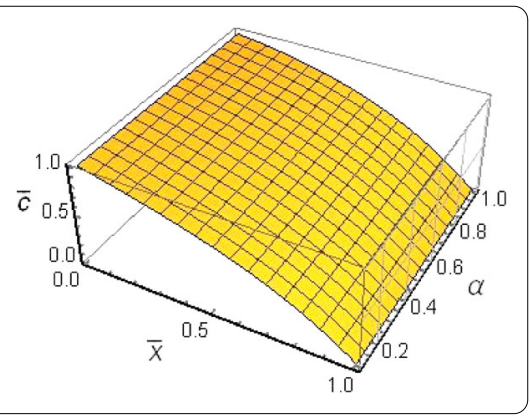

Figure 7 Dependence of the solution (40) on distance $\bar{x}$ and the time parameter $\kappa(\bar{v}=2, \alpha=0.5)$.

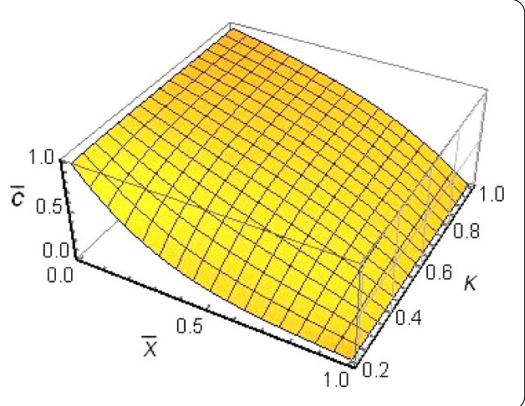

Figure 8 Dependence of the solution (40) on distance $\bar{x}$ and the drift parameter $\bar{v}(\alpha=0.5, \kappa=0.2)$.

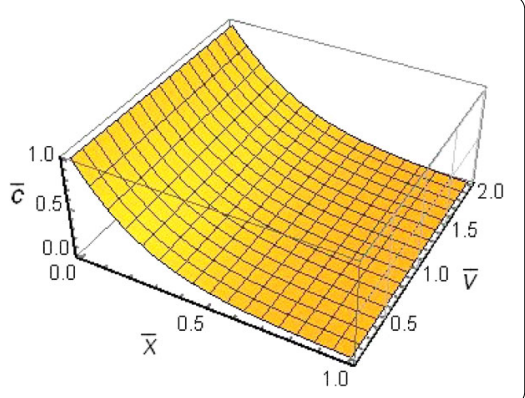

\section{Conclusions}

We have considered the time-fractional advection-diffusion equation with the Caputo fractional derivative in a domain $0<x<L$. The Laplace transform with respect to time $t$ and the finite sin-Fourier transform with respect to the spatial coordinate $x$ have been used. The fundamental solution to the Dirichlet problem and the solution to the problem with a constant boundary condition for the sought-for function have been obtained. The results of numerical calculations are displayed in the figures for different values of the 
nondimensional spatial variable $\bar{x}$, the drift parameter $\bar{v}$, the time parameter $\kappa$, and the order of the time-fractional derivative $\alpha$. To evaluate the Mittag-Leffler functions $E_{\alpha, \alpha}(-x)$ and $E_{\alpha}(-x)$ we have used the algorithms suggested in [28] (the interested reader is also referred to the Matlab programs that implement these algorithms [29]). It should be emphasized that the first term in curly brackets in the solution (39) satisfies the boundary condition (31) and (32), whereas the second one equals zero at the ends of a line segment $0<x<L$ due to (19).

\section{Competing interests}

The authors declare that they have no competing interests.

\section{Authors' contributions}

Each of the authors contributed to each part of this work equally and read and approved the final version of the manuscript.

\section{Author details}

${ }^{1}$ Institute of Mathematics and Computer Science, Jan Długosz University, Częstochowa, 42-200, Poland. ${ }^{2}$ Institute of Mathematics, Czȩstochowa University of Technology, Czȩstochowa, 42-200, Poland.

Received: 11 June 2015 Accepted: 21 April 2016 Published online: 28 April 2016

\section{References}

1. Kaviany, M: Principles of Heat Transfer in Porous Media, 2nd edn. Springer, New York (1995)

2. Feller, W: An Introduction to Probability Theory and Its Applications, 2nd edn. Wiley, New York (1971)

3. Scheidegger, AE: The Physics of Flow Through Porous Media, 3rd edn. University of Toronto Press, Toronto (1974)

4. Van Kampen, NG: Stochastic Processes in Physics and Chemistry, 3rd edn. Elsevier, Amsterdam (2007)

5. Risken, H: The Fokker-Planck Equation. Springer, Berlin (1989)

6. Nield, DA, Bejan, A: Convection in Porous Media, 3rd edn. Springer, New York (2006)

7. Metzler, R, Klafter, J: The random walk's guide to anomalous diffusion: a fractional dynamics approach. Phys. Rep. 339, 1-77 (2000)

8. Rossikhin, Y, Shitikova, MV: Applications of fractional calculus to dynamic problems of linear and nonlinear hereditary mechanics of solids. Appl. Mech. Rev. 50, 15-67 (1997)

9. West, BJ, Bologna, M, Grigolini, P: Physics of Fractal Operators. Springer, New York (2003)

10. Magin, RL: Fractional Calculus in Bioengineering. Begell House Publishers, Inc., Redding (2006)

11. Gafiychuk, V, Datsko, B: Mathematical modeling of different types of instabilities in time fractional reaction-diffusion systems. Comput. Math. Appl. 59, 1101-1107 (2010)

12. Mainardi, F: Fractional Calculus and Waves in Linear Viscoelasticity: An Introduction to Mathematical Models. Imperial College Press, London (2010)

13. Uchaikin, W: Fractional Derivatives for Physicists and Engineers, Background and Theory. Springer, Berlin (2013)

14. Povstenko, Y: Fractional Thermoelasticity. Springer, New York (2015)

15. Povstenko, Y: Theory of diffusive stresses based on the fractional advection-diffusion equation. In: Abi Zeid Daou, R, Moreau, X (eds.) Fractional Calculus: Applications, pp. 227-241. Nova Science Publishers, New York (2015)

16. Povstenko, Y: Fractional heat conduction equation and associated thermal stress. J. Therm. Stresses 28, 83-102 (2005)

17. Povstenko, Y: Thermoelasticity which uses fractional heat conduction equation. J. Math. Sci. 162, 296-305 (2009)

18. Povstenko, Y: Theory of thermoelasticity based on the space-time-fractional heat conduction equation. Phys. Scr. T $136,014017(2009)$

19. Povstenko, Y: Non-axisymmetric solutions to time-fractional diffusion-wave equation in an infinite cylinder. Fract. Calc. Appl. Anal. 14, 418-435 (2011)

20. Gorenflo, R, Mainardi, F: Fractional calculus: integral and differential equations of fractional order. In: Carpinteri, A, Mainardi, F (eds.) Fractals and Fractional Calculus in Continuum Mechanics, pp. 223-276. Springer, Wien (1997)

21. Podlubny, I: Fractional Differential Equations. Academic Press, New York (1999)

22. Kilbas, AA, Srivastava, HM, Trujillo, JJ: Theory and Applications of Fractional Differential Equations. Elsevier, Amsterdam (2006)

23. Povstenko, Y: Fundamental solutions to time-fractional advection diffusion equation in a case of two space variables. Math. Probl. Eng. 2014, 705364 (2014)

24. Liu, F, Anh, V, Turner, l, Zhuang, P: Time-fractional advection-dispersion equation. J. Appl. Math. Comput. 13, 233-245 (2003)

25. Huang, F, Liu, F: The time fractional diffusion equation and the advection-dispersion equation. ANZIAM J. 46, 317-330 (2005)

26. Huang, F, Liu, F: The fundamental solution of the space-time fractional advection-dispersion equation. J. Appl. Math. Comput. 18, 339-350 (2005)

27. Prudnikov, AP, Brychkov, YA, Marichev, Ol: Integrals and Series. Vol. 1: Elementary Functions. Gordon \& Breach, Amsterdam (1986)

28. Gorenflo, R, Loutchko, J, Luchko, Y: Computation of the Mittag-Leffler function and its derivatives. Fract. Calc. Appl. Anal. 5, 491-518 (2002)

29. Matlab File Exchange 2005, Matlab-Code that calculates the Mittag-Leffler function with desired accuracy. Available for download at www.mathworks.com/matlabcentral/fileexchange/8738-Mittag-Leffler-function 